
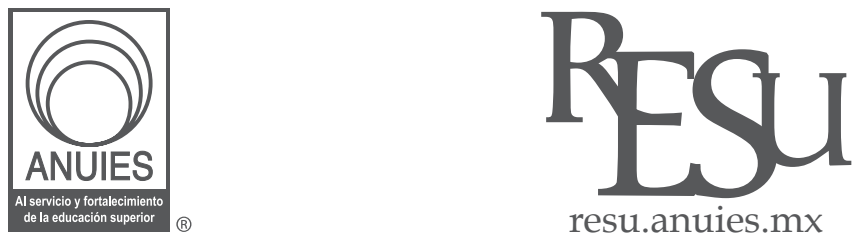

ARTí́CULO

\title{
La Educación Superior como autoformación: el caso de estudiantes transfronterizos*
}

\author{
Simon Marginson** \\ * Título original: Higher education as self-formation: the case of cross-border students. (Fátima Rateb, fatimarateb@ \\ gmail.com). Versión original en inglés en http://resu.anuies.mx \\ ** Profesor de Educación Superior Internacional en el Instituto de Educación de la Universidad de Londres en el \\ Reino Unido. Es editor adjunto de la revista Higher Education y autor principal del libro International Student Security \\ (2010) publicado por Cambridge University Press.
}

Recibido el 23 de noviembre del 2013; aprobado el 4 de diciembre del 2013

\section{PALABRAS CLAVE}

Estudiantes internacionales/

Globalización/Adaptación/

Adecuación cultural/

Identidad/Autoformación

\section{Resumen}

En la investigación sobre la psicología multicultural, la educación internacional transfronteriza se entiende como un proceso de "adaptación" a las normas e instituciones del país anfitrión. El estudiante se considera deficitario con respecto a estos requerimientos y la identidad del país de origen se convierte en una barrera que se debe abatir. Sin embargo, este artículo define la educación internacional

como un proceso de autoformación en el que los sujetos-estudiantes gestionan sus vidas de manera reflexiva, moldeando sus propias identidades, aunque dentro de condiciones y relaciones sociales no creadas por ellos en gran medida. Ellos dan forma tanto a su "yo" como a sus trayectorias entre la identidad del país de origen, la identidad del país anfitrión y un conjunto más amplio de opciones cosmopolitas.

\section{KEYWORDS}

International students/

Globalization/Adjustment/

Cultural fit/Identity/

Self-formation

\begin{abstract}
In research in cross-cultural psychology, cross border international education is largely understood as a process of 'adjustment' to host country norms and institutions. The student is seen as in deficit in relation to these requirements. Home country identity becomes a barrier to be broken down. This paper instead defines international education as a process of self-formation in which student subjects
\end{abstract} manage their lives reflexively, fashioning their own changing identities, albeit within social conditions and relations largely not made by them. International students form their selves and their trajectories between home country identity, host country identity and a larger set of cosmopolitan options.

Para la investigación de este artículo se recibió el apoyo del Consejo Australiano de Investigación (Australian Research Council) y de la Universidad de Monash en Australia. 


\section{LA EDUCACIÓN SUPERIOR COMO AUTOFORMACIÓN}

\section{Introducción}

$\mathrm{E}$ ste artículo surge de las reflexiones hechas a lo largo de casi una década de investigación sobre el bienestar y la seguridad de los estudiantes (Sawir, Marginson, Deumert, Nyland y Ramia, 2008; Marginson, Nyland, Sawir y Forbes-Mewett, 2010), las relaciones multiculturales en la educación internacional (Marginson y Sawir, 2011) y las políticas y regulaciones en la educación internacional (Marginson, 2012; Ramia, Marginson y Sawir, 2013). Se incluyeron entrevistas semi-estructuradas con una duración de 30 a 60 minutos a 290 sujetos-estudiantes internacionales que estudian en Australia y Nueva Zelanda, cuatro quintas partes provenientes del este, el sudeste y el sur asiático.

Más de dos terceras partes de los estudiantes entrevistados no tenían familiares cercanos en el nuevo lugar de residencia, nueve de diez recibían apoyo de su familia, algunos tenían a sus cónyuges y/o hijos con ellos y muy pocos vivían con sus familias biológicas. Casi todos eran independientes de manera cotidiana, físicamente alejados de sus orígenes y labrando su propio camino en el mundo. Esto condujo a efectos contrastantes. Por un lado, casi dos tercios de los estudiantes reportaron sentirse solos y/o aislados en un momento dado durante su estancia en el extranjero. Una minoría dijo que la sensación de soledad persistió después de los primeros seis meses (Marginson, et al., 2010: 338-343 y 376-382; Sawir, et al., 2008). Por otro lado, la independencia trajo consigo la sensación de libertad como autodeterminación; no tanto como libertinaje sin restricciones, sino como libertad de hacer y de ser.

Todos los estudiantes internacionales cruzan las fronteras para ser diferentes: sea a través del aprendizaje, para obtener un título universitario, por la inmersión en el entorno lingüístico o simplemente para su crecimiento personal. A menudo anhelan convertirse en cierto tipo de persona, aunque ninguno puede imaginarse completamente el resultado previo a la transformación. Algunos responden al cambio sólo cuando deben hacerlo, muchos dejan que suceda por sí solo, mientras otros corren a su encuentro. Esta experiencia de la agencia ${ }^{1}$ autodirigida durante la estadía en el extranjero -las alegrías y los miedos de la construcción del yo en medio de una cantidad de opciones frecuentemente novedosas- es poco reconocida y, sin embargo, es un sentimiento generalizado. Como lo expresaron dos estudiantes:

Hay muchísimas diferencias entre la vida de allá y la de aquí. La ventaja de vivir aquí es que te enseña a ser independiente, es la supervivencia del más apto; cómo hacer las cosas y administrar toda tu vida. En casa tienes a tus padres como apoyo. Aquí no hay apoyo, estás por tu cuenta. Existen decisiones cruciales y tienes que tomar esas decisiones,

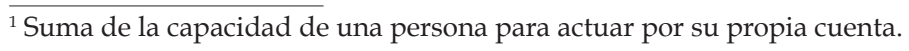


no tus padres. Aprendes mucho.

(Hombre, 27 años, estudios empresariales).

Algunos vivieron esta sensación del yo independiente como liberadora:

Me encanta este lugar, me siento cómoda. ¿Sabes?, encajo aquí. En la India no encajo, soy feminista. Es verdad, soy una mujer de carácter fuerte y en el subcontinente indio es muy difícil. [Aquí] tengo la libertad de vivir mi propia vida y no tengo que regresar a casa y... No tengo que lidiar con todas esas presiones sociales. Tengo mi vida.

(Mujer, 19 años, artes)

Este prolongado compromiso con estudiantes internacionales ha cuestionado la manera en la que se estructura la educación internacional en la investigación social. Este artículo argumenta a favor de un cambio de paradigma, desde entender la educación internacional como un proceso de "adaptación" de estudiantes extranjeros a requerimientos locales -el paradigma que actualmente predomina es la investigación sobre estudiantes internacionales- para entender la educación internacional como una autoformación. Esto proporciona una perspectiva sobre la identidad y la agencia, explora posibilidades y límites de la autoformación y considera las estrategias usadas por los estudiantes. No se basa en pruebas ilustrativas de índole empírica, se trata esencialmente de una teorización.

\section{El paradigma de adaptación en la psicología}

En la psicología multicultural la educación internacional se entiende principalmente como un proceso de "adaptación" o "aculturación" a los requerimientos y los hábitos del país anfitrión. Aunque uno de los primeros psicólogos multiculturales más influyentes señaló que el paradigma de adaptación no era esencial para el campo de conocimiento (Bochner, 1972), en el que era ampliamente utilizado, en Church (1982) encontramos una de las primeras discusiones superiores dentro del paradigma. Volet y Jones (2012), por su parte, proporcionan un análisis crítico del paradigma que complementa el presente artículo.

En el paradigma de la adaptación se piensa que la educación internacional es como un viaje mutuo entre la cultura del país de origen y la del país anfitrión, facilitado por el dominio del idioma y la participación multicultural. Cada cultura es asumida como una constante y los estudiantes internacionales avanzan en las instituciones anfitrionas al adquirir los atributos locales necesarios para el bienestar psicológico y el éxito académico. La cultura del país anfitrión se normaliza, las instituciones del país anfitrión se aceptan como son y el estudiante internacional se considera en déficit con respecto a los requerimientos del país anfitrión. Los estudios individuales varían de acuerdo con el grado en el que se contemplan las nociones de identidades múltiples, algunos no consideran patológico el compromiso 
continuo a la cultura del país de origen, pero el análisis cuantitativo funciona mejor con nociones fijas, delimitadas y, si es posible, de identidad. Y el punto final de la narrativa es la asimilación. En este trabajo, la identidad del país de origen frecuentemente es vista como un fenómeno o un obstáculo que se debe abatir.

El paradigma de adaptación se traduce muy fácilmente al sentido de superioridad cultural que -hay que decirlo- abunda en los sistemas de educación de habla inglesa (Lee y Rice, 2007; Marginson et al., 2010; Montgomery, 2010). Después de todo, sigue la lógica etnocéntrica, ¿por qué otra razón se inscribirían estudiantes internacionales en nuestras instituciones, si no para ser como nosotros? Se da por supuesto que los psicólogos multiculturales y los educadores de los países anfitriones conocen a los estudiantes mejor que a ellos mismos, y saben en lo que se tienen que convertir. En pedagogías informadas por este enfoque, la agencia del estudiante internacional no es totalmente suprimida, más bien, el objetivo es rehacer la agencia del estudiante cambiándole de forma para "vaciar" esos hábitos y valores anteriores, considerados obstáculos, e (irónicamente) instalar en el estudiante extranjero un aprendiz autónomo "occidental" imaginario (Doherty y Singh, 2005. Un texto similar que todavía se usa mucho en la capacitación de docencia universitaria es Ballard y Clanchy, 1991).

Esta situación causa molestias en los estudiantes internacionales, quienes expresan su deseo de adquirir aquellos atributos locales necesarios para ser exitosos, no se niegan a recibir consejos y muchas veces están impresionados con lo que ven en el país anfitrión, sin la necesidad de abandonar su yo ni la identidad formada en el país de origen para lograr aculturizarse.

Una rama de la investigación que engloba estas limitaciones es el trabajo sobre la "adecuación cultural" de Ward y sus colegas (Marginson y Sawir, 2011: 30-48), quienes opinan que mientras más se asemeja la cultura entre los estudiantes y la institución anfitriona, es más probable que los estudiantes se "adapten" exitosamente y progresen académicamente (Ward y Chang, 1997; Leong y Ward, 2000). En ella se trabaja con nociones esencialistas de identidad cultural, como el contraste de Hofstede (1998) entre las culturas "individualistas" y "colectivistas".

Sin embargo, la idea de "adecuación cultural" falla repetidamente en las pruebas empíricas usadas en el cuerpo de este trabajo. Los estudiantes que se determinaron como culturalmente alejados del país anfitrión no necesariamente tuvieron un menor desempeño o presentaron niveles de bienestar inferiores. La identidad cultural no está necesariamente relacionada con el éxito académico (Li y Gasser, 2005) -las relaciones suceden por muchos otros factores- y los estudiantes internacionales no abandonan las identidades de su país de origen, incluso la identidad de "herencia" sigue cambiando en el país anfitrión, donde los estudiantes mezclan identidades en maneras complejas y variables, muchas veces deliberadamente (Kashima y Loh, 2006). El fracaso empírico de la hipótesis de la "adecuación cultural", cabe destacar, no ha disuadido a sus partidarios de continuar sosteniéndola, lo cual demuestra 
la resistencia de las conjeturas iniciales (Ward, Leong y Low, 2004; Yang, Noels y Saumure, 2006).

El elemento subestimado en los trabajos sobre "adecuación cultural", y en la mayoría de los estudios sobre adaptación, es la agencia activa de los mismos estudiantes internacionales, lo cual no se debe exclusivamente al etnocentrismo, se trata también de un problema metodológico. No todos los estudios psicológicos sobre la educación internacional son etnocéntricos y algunos se refieren a la identidad como abierta y plural, como más adelante presentaremos. No obstante, es difícil entender las identidades humanas cambiantes, la imaginación, la agencia, la reflexividad y la auto-creación, utilizando métodos de análisis de regresión que predominan en la corriente principal de la psicología multicultural. Los métodos cualitativos son menos precisos pero más incluyentes, las entrevistas semi-estructuradas permiten que los sujetos-estudiantes contribuyan al desarrollo conceptual, por ejemplo, aportando perspectivas e ideas nuevas al campo de investigación. Se habla mucho de que la educación "gravita" en torno al estudiante, pero la idea de la educación internacional como autoformación, no la adaptación dirigida a otros estudiantes, se sustenta en torno a las prácticas del estudiante mismo y esto tiene implicaciones tanto metodológicas como normativas.

\section{Agencia e identidad}

¿Cuáles son, entonces, los elementos candidatos para un enfoque alternativo de la identidad y de la agencia de los estudiantes internacionales, en donde estos últimos son considerados principalmente como auto-formados en vez de "adaptados"? La teorización que prosigue se basa en parte en Sen (1985, 1992, 1999, 2000), cuyo interés central está en la capacidad humana y en el contexto de la pluralidad cultural.

La identidad es ambigua: es cómo nos llamamos y cómo nos llaman los otros. Bourdieu (1993: 30) habla de sujetos humanos que se autoposicionan y, al mismo tiempo, son posicionados dentro de un "espacio de posibilidades" socialmente constituido. Mientras todas las relaciones humanas son construidas socialmente y limitadas, la noción de autoformación utilizada aquí es más optimista que Bourdieu con respecto a la apertura y la autonomía. Los sujetos eligen la movilidad para cambiar su espacio de posibilidades y los estudiantes internacionales crean una mezcla de identidad a partir de un abanico más amplio de opciones definidas socialmente. Sen hace hincapié en que "todos tenemos identidades múltiples" y nuestras diferentes identidades están asociadas a preocupaciones distintivas y, a veces, en conflicto. Además de la compartida identidad humana y de la particular identidad nacional, las personas también se identifican de diversas formas en términos de religión, clase social, género, localidad, familia, política, "identidades profesionales" -como doctor o educador-, entre otros (Sen, 1999: 116-125). Cada vez más, a través 
de la comunicación mundial y la movilidad, las identidades cruzan las fronteras nacionales.

Esto no significa que la identidad esté fragmentada como un sistema sin características en el que cada elemento es intercambiable con cualquier otro, la identidad se constituye como un campo de diferencia en el que algunas identidades son primarias. En las 290 entrevistas, muchos estudiantes internacionales vieron ciertos elementos de autoidentidad como un cambio lento: elementos vinculados con las relaciones de familia, o con la identidad cultural o nacional, o el primer idioma hablado, o los recuerdos definidos como "casa" o "el verdadero yo". Las personas necesitan tanto la autodefinición como la capacidad de reaccionar ante el cambio. Paradójicamente, este aspecto de singular certeza, con sus significantes más duraderos del yo, es una de las condiciones que permite la flexibilidad y la pluralidad.

Sin embargo, una taxonomía de la identidad sólo nos acerca un poco a la autoformación. La identidad no consiste en la persona entera, sino en un manto que usa y que se puede cambiar en otro momento. Existe un lado delicado en la identidad, una cierta inflexibilidad que paraliza cualquier posibilidad de movimiento, nosotros evolucionamos, no así las etiquetas que elegimos. Aunque estas etiquetas pueden adoptar diferentes matices en su significado, están vinculadas al uso común, un lastre poco elástico. Las personas necesitan que la identidad les garantice seguridad y certeza, pero las etiquetas no sustituyen la descripción holística de la persona ni sus características relacionales. La identidad sólo es una herramienta que las personas usan cuando se forman, sólo es uno de los aspectos con el cual los estudiantes internacionales (y cualquier otra persona) pueden ser percibidos.

\section{Libertad de agencia}

¿Cómo podríamos entender a la persona en el proceso de autoformación dado que la identidad por sí sola no es suficiente? Si la identidad es lo que una persona entiende que él o los otros son, la agencia es la suma de la capacidad de una persona para actuar por su propia cuenta. Mientras que las etiquetas de identidad de una persona son elegidas (por ellos mismos) o impuestas por instituciones sociales (o de investigación), la agencia de esa persona es irreductible. Sen presenta un caso persuasivo de libertad como autodeterminación: un "agente" es "alguien que actúa y propicia el cambio, y cuyos logros pueden ser juzgados en términos de sus propios valores y objetivos, sin importar si los evaluamos en términos de criterios externos también [...]", "Los adultos responsables deben estar a cargo de su propio bienestar; en ellos está decidir cómo usar sus capacidades" (Sen, 2000: 19). Agrega que las capacidades de una persona "dependen de la naturaleza de los acuerdos sociales, lo que puede ser crucial para las libertades individuales" (Sen, 2000: 288). En relación con los estudiantes internacionales en educación superior, el primer 
paso para comprender su autoformación es verlos como adultos responsables, en el sentido que describe Sen, y no tanto como niños dependientes.

El concepto de Sen de la libertad humana incluye tres elementos. En primer lugar, la libertad del individuo con respecto a amenazas externas, coerción o restricciones -Sen le llama "libertad de control" y corresponde grosso modo a la "libertad negativa" en Isaiah Berlin o F.A. Hayek-. En segundo lugar, la libertad como capacidad del individuo para actuar, lo que depende de capacidades y recursos, y de acuerdos sociales que permiten que las personas pongan en práctica sus elecciones. Sen llama a esto "la libertad como poder" y en trabajos posteriores, "libertad efectiva"; otros lo llaman libertad positiva (Sen, 1985, 1992). En tercer lugar, la "libertad de agencia", la voluntad humana activa, la sede de acciones conscientes autodirigidas, que guían la autoformación reflexiva y la autonegociación de la identidad. Los tres elementos de la libertad son interdependientes, la libertad de control y la libertad efectiva pueden entenderse como momentos defensivos y proactivos de la agencia humana, mientras que la libertad de agencia ocupa el papel central en la concepción de la libertad de Sen.

Sen señala que las perspectivas de "bienestar" y de "agencia" producen diferentes nociones de libertad (Sen, 1985: 169). La noción de bienestar sugiere un individuo que toma decisiones pero no necesariamente significa que sea activo o interactivo; a diferencia de la noción de agencia que sugiere una voluntad humana intrínsecamente proactiva. En la perspectiva de bienestar, se considera a la persona como un beneficiario cuyos intereses y ventajas son lo más importante, mientras que en la perspectiva de agencia, la persona es considerada como un emprendedor y un juez, con distintas implicaciones para las metas y valuaciones de la persona. “El aspecto de bienestar de una persona es importante en la evaluación de la venta$j a$, mientras que el aspecto de agencia es importante al evaluar lo que una persona puede hacer en consonancia con su concepción del bien", que no tiene por qué ser ventajoso para la persona (Sen, 1985: 206, con énfasis en el original). La perspectiva de bienestar ha llamado más la atención que la de agencia, Sen no lo dice, pero esto puede ser el fruto del utilitarismo y de la economía neoclásica. No obstante, el bienestar económico no es cimiento suficiente para la libertad. La libertad de agencia va más allá del cálculo económico para incluir estatus, dignidad, familia, amigos, hacer cosas, un trabajo que satisfaga y el alcance para realizar ciertas formas de vida. Los bienes colectivos compartidos son importantes, al igual que los bienes individuales. Los agentes humanos en autoformación eligen lo que quieren a partir de ver los "menús" y sus posibilidades.

En la educación internacional transfronteriza, en donde los estudiantes visualizan el viaje antes de ver el terreno, no todos los sueños iniciales pueden realizarse. Los estudiantes se adaptan, así como su trayectoria de predilección, conforme avanzan. Algunas personas imaginan lo imposible para sí mismos y más adelante cambian sus nociones del bien. 


\section{La educación internacional como autoformación}

La educación superior como autoformación va más allá de la noción del estudiante como consumidor de un mercado; se asemeja más a criar niños que, por ejemplo, a fabricar y vender lavadoras. Implica invertir en sí como capital humano porque los atributos y las cualificaciones adquiridas en la educación cambian lo que puede hacer una persona. Para los estudiantes internacionales transfronterizos, más que para la mayoría de los estudiantes, los costos son elevados. Los frutos económicos de la inversión importan y, sin embargo, en ese proceso de transformación no sólo buscan una carrera o mejores ingresos.

La educación internacional como autoformación incorpora un conjunto más extenso de comportamientos para cultivarse y superarse personalmente: aprender a hablar en nuevos lenguajes conversacionales, la adquisición de conocimientos y sensibilidades personales mediante la educación liberal como capital cultural, e incorporar capital social a través del cual la educación superior fomenta relaciones funcionales y redes sociales. Esto explica el hecho de que los estudiantes pueden adquirir nuevos valores y creencias en el país de educación, y tal vez mayor tolerancia y más relaciones cosmopolitas (Montgomery, 2010). Aquí, el incremento de las capacidades individuales y el aumento de sociabilidad son interdependientes. Los diferentes efectos de la educación superior son considerados como en tensión. Por ejemplo, la educación superior como inversión en la capacidad de obtener ingresos se contrapone a la educación para adquirir conocimientos o autoconocimientos, como si los dos aspectos no pudieran coexistir; sin embargo, muchos estudiantes quieren ambas cosas. Los diferentes efectos heterogéneos encuentran refugio bajo la autoformación, cada uno de los programas liberales y de formación profesional contribuye en la construcción reflexiva del yo de la persona y al ensamblaje de sus habilidades, conocimientos, talentos, hábitos y aspiraciones.

La educación internacional como autoformación también significa que en lugar de que el estudiante internacional sea visto habitualmente como débil o deficiente, se considere como un agente fuerte que dirige el curso de su vida. Si bien todos los estudiantes de educación superior -como todos los sujetos humanos- están en continua autoformación, el papel de la agencia en el autocambio en los estudiantes internacionales es particularmente evidente. En primer lugar, su situación los obliga a cambiar de manera dinámica, como se ha señalado, en su mayoría tienen una gran autonomía práctica, en el sentido de que viven lejos de su familia biológica; especialmente al principio, adquieren nuevos atributos en sus estudios, sus relaciones institucionales y sus vidas cotidianas. La autoformación no sigue los modelos de equilibrio de la psicología. De hecho, los estudiantes internacionales buscan desequilibrios: quieren ser diferentes y muchas veces los más rápido posible (esto contrasta con Ramsay, Barker y Jones, 1999: 130, quienes conciben la "adaptación" de estudiantes internacionales como la eliminación sucesiva de "insatisfactores psicológicos" generando "desequilibrios"). Esta ambición, cuando menos, se extiende 
a la adquisición tanto del dominio del idioma inglés, como de las capacidades académicas y de algunos amigos locales. A medida que la estancia transcurre, muchos cambian su forma de vida, patrones de consumo y hasta creencias personales.

Las posibilidades no deben ser idealizadas, aunque los estudiantes están en el camino que eligieron y que los resultados pueden ser valiosos, su autoformación en el día a día se viste mayoritariamente de necesidad, sobrevivencia y enfrentamiento, en lugar de ser una aventura voluntaria. Rizvi (2009: 261) observa que las transformaciones prometidas son "inherentemente contradictorias" y la gente se debate, por un lado, entre "la flexibilidad cultural", y por el otro la "incertidumbre y confusión cultural". No todos los estudiantes internacionales alcanzan plena confianza en sí mismos, como siempre sucede con los fenómenos mundiales, tanto las posibilidades como los resultados están distribuidos desigualmente. Los estudiantes internacionales se forman bajo condiciones sociales que no pueden controlar, que moldean su "espacio de posibilidades" y difieren de un estudiante a otro; algunos tienen más recursos, otros son más vulnerables, otros más tienen atributos personales que se integran fácilmente al país de educación. Para muchos, es un ambiente poco familiar y en ocasiones hostil, la comunicación en el día a día es difícil, las curvas de aprendizaje son empinadas y la cima fuera de su alcance. Muchos otros buscan una interacción social intensiva con estudiantes locales, pero estos últimos rara vez corresponden a ese anhelo (Marginson, et al., 2010: capítulos 13-15). La educación internacional en los países de habla inglesa contribuye poco a la autoformación de la mayoría de los estudiantes locales como ocurre en otros lugares. Estos bloqueos y turbulencias ponen a los estudiantes internacionales en ciertos caminos y no en otros. El estudiante internacional no tiene el control de su propio destino. Sin embargo, incluso cuando el estudiante se encuentra aislado, bajo el mando de otros, o atrapado entre dos grupos conflictivos, ejerce mayor autonomía que otras personas.

Además de su carácter integral y agencial, la educación internacional como autoformación es distintiva de otras, y como toda autoformación, está históricamente arraigada y sujeta a relaciones de poder.

La autoformación de estudiantes internacionales es abierta no sólo porque la libertad de agencia lo permite, sino porque los entornos institucionales y culturales exigen cosas que no se conocen antes de haberlos vivido y a los cuales ellos deben responder. Bajo condiciones nuevas, la gente hace cosas nuevas; en comparación con los estudiantes locales, la mayoría de los internacionales se enfrentan a posibilidades más amplias y variadas, mientras que el ambiente de vida de las personas no móviles es conocido en su mayoría. Los estudiantes internacionales construyen esos ambientes de vida en términos de las infraestructuras físicas, dónde viven y de muchas de sus pertenencias personales e infraestructuras sociales, de sus relaciones formales e informales. La educación internacional no sólo ofrece problemas y barreras sino también oportunidades de actividades novedosas y de crecimiento personal. Por ejemplo, la ausencia de enlaces afectivos cercanos abre caminos 
para nuevas amistades con posibilidades desconocidas de aprendizaje y riesgos. También existe la posibilidad de combinar culturas en la forma que elijan. Muchos estudiantes lo hacen de manera explícita, hablando de selección cultural entre sí, acceden a culturas de origen, culturas anfitrionas y las de los nuevos conocidos extranjeros, con quienes es más fácil establecer amistades que con los locales (Kashima y Loh, 2006).

La autoformación de estudiantes es compleja porque implica más de un proyecto -educativo, económico, ocupacional, familiar, cultural, social, lingüístico, etcétera- y no se puede reducir a un indicador en una escala común. Para comprender la autoformación de un estudiante internacional es necesario sintetizar estos diferentes elementos y sólo se puede hacer a través de actos de juicio complejo. Sin embargo, los estudiantes en forma individual emiten un juicio muy holístico sobre sí mismos de manera continua; el proceso es profundamente reflexivo. En la autoformación, la gente aprende a moldearse conforme pasa el tiempo, muchas veces conscientes de sus propias y cambiantes subjetividades, sus observaciones son muy críticas desde un punto de vista personal y de los demás. Los estudiantes internacionales tienen dificultades en convertirse en lo que ellos quieren ser. Muchas veces, los resultados son diferentes de lo que desean o esperan. Pero no se dan por vencidos, pues remodelan sus intenciones a medida que avanzan, oscilando entre luchar contra lo que ven como sus propias deficiencias, aceptar temporalmente las limitaciones y empujar hacia delante de nuevo. En muchas ocasiones, los agentes reflexivos identifican y desafían sus propias suposiciones (Rizvi, 2008: 33). Durante las entrevistas no todos los estudiantes internacionales dan su opinión sobre su propia evolución reflexiva y su cambiante identidad, pero muchos otros sí.

La autoformación se ve afectada por los tiempos y lugares donde ocurre. Ese yo en constante movimiento está activo en más de un lugar y tiempo, conectado al país de origen a través de los medios de comunicación, como señala Appadurai (1996). Se toca el tema de la autoformación en todas las instituciones, ambientes profesionales, lugares públicos, grupos de actividades y entornos privados; son moldeadas por todos los lugares en donde la identidad de las acciones está involucrada, en encuentros multiculturales, en economía material, en políticas y regulaciones, y en otras relaciones de poder. Los estudiantes internacionales de color, que estudian en países de habla inglesa, a menudo sufren discriminación o abuso que limitan el potencial de autoformación, al desalentar una mayor integración en la sociedad anfitriona (Lee y Rice, 2007; Marginson, et al., 2010: capítulo 15).

\section{Estrategias para la autoformación}

En resumen, los estudiantes transfronterizos cambian sus circunstancias, sus caminos y sus posibilidades. Su autoformación es continua, ese ímpetu está sustentado en la memoria, la observación, la experiencia y por la formación consciente del 
yo, como un proyecto personal, típico en la vida de aquéllos que han recibido una educación (Rose, 1999). En el país anfitrión, el estudiante transfronterizo se forma a sí mismo usando tanto recursos que trae consigo como aquéllos que encuentra ahí, donde los estudiantes se abren a la cultura anfitriona, hasta aquel punto donde es necesario para sobrevivir.

La autoformación es un trabajo de la imaginación en el que las posibilidades son configuradas mediante la coordinación de más de un grupo cultural. Una característica distintiva de la estancia de un estudiante internacional es estar consciente de las muchas posibilidades y ejemplos que tiene para construir el yo. En su mayoría, la autoformación de un estudiante en esta pluralidad cultural está configurada por lo que llamaremos multiplicidad e hibridez, con la premisa que pueden tomar una o ambas. La psicología y la sociología multicultural aluden frecuentemente a estos dos métodos o estrategias para gestionar la autodefinición y el desarrollo.

\section{Multiplicidad}

Es la primera estrategia de la autoformación, donde el estudiante extranjero se considera como algo más que una persona viviendo más de una vida. Berry (1974) en sus primeros trabajos, sugiere la existencia de un yo bicultural en el que conviven dos diferentes identidades, con énfasis variable en una u otra. Kashima y Loh (2006) señalan que más de dos conjuntos culturales son posibles. Muchas veces, esa línea divisora está planteada por el idioma que usan. El estudiante es una persona algo diferente en el entorno del país anfitrión, puesto que usa el idioma y las referencias culturales de ese país. Pedersen (1991: 22) piensa que este tipo de multiplicidad es común y sugiere que la identidad consta de una capa superior y otra inferior, semejante a una excavación arqueológica. El estudiante internacional mantiene creencias y prácticas de su país de origen en ámbitos como la familia, el matrimonio y la religión, luego agrega una capa de un conjunto de nuevas prácticas cotidianas, facilitando así asociaciones en el país anfitrión. El estudiante también adquiere un mayor sentido del relativismo cultural y una mayor reflexividad con un acercamiento más consciente y deliberativo a las elecciones personales y a la formación de la identidad. Las personas que son biculturales de nacimiento tienden a practicar una mezcla cultural de manera más constructiva y con menor esfuerzo (Volet y Ang, 1998: 8).

Berry imagina la identidad bicultural en términos de narrativa lineal, un viaje desde la salida del país de origen hasta el destino en el país anfitrión. La oscilación está entre el pasado y el presente, como si la memoria y la experiencia no convivieran cotidianamente y la memoria no se reinterpretara continuamente, donde el contacto cultural con "la casa" no se mantuviera y la identidad patrimonial no fuera un yo vivo. Más bien, como señala Butcher (2002) en un estudio de estudiantes graduados después de su regreso a casa, existen muchas posibles configuraciones 
multiculturales de la identidad. A pesar de las relaciones dominantes con las que se expresan muchas veces las culturas del país anfitrión, el mantenimiento y la adaptación cultural no necesariamente están en oposición (Lee y Koro-Ljungberg, 2007). Esto nos lleva a la segunda estrategia de la autoformación: la hibridez.

\section{Hibridez}

El estudiante internacional sintetiza diferentes elementos culturales y relacionales en un nuevo yo. Al final de la estancia, en lugar de regresar a una identidad anterior, de su país de origen, se lleva a casa un yo transformado. Rizvi (2005: 336) ve la hibridez como "un espacio en el que debemos aprender a manejar incertidumbres culturales". En lugar de vivir las culturas como algo holístico, "prístino y auténtico", éstas se contaminan entre sí. Cuando los estudiantes viajan, "las formas culturales se separan de las prácticas existentes y se recombinan con nuevas formas". En un mundo constituido por "flujos de dinero, tecnología y gente, a través del turismo, la educación y migración", la hibridación se ha convertido en "una condición de existencia social", y no es "algo excepcional".

Como la multiplicidad, la hibridez está asociada a una mayor reflexividad y a un sentido de relativismo cultural. La apertura a los otros es esencial. Existen pocos puntos finales o resoluciones claras. Goldbart y sus colegas (2005: 105) consideran la educación superior como una "zona de contacto" en la que diferentes culturas "luchan contra sí" en condiciones de poder desigual. Las culturas rara vez se combinan simétricamente. Rizvi advierte que mientras la hibridez es "un antídoto útil contra el esencialismo cultural", la hibridez sola no explica las relaciones culturales, pues sigue siendo necesario investigar "cómo la hibridez ocurre, la forma que toma en contextos específicos, las consecuencias que tiene para determinados sectores de la comunidad y cuándo y cómo ciertas formaciones híbridas son progresivas o regresivas" (Rizvi, 2005: 338).

Cada estrategia se puede describir por metáforas espaciales (aunque tienen sus límites). La multiplicidad está asociada a dividir o diferenciar. La hibridez está asociada a integrar, suturar, combinar o recombinar. Aquí, dividir y combinar son dos lados de la moneda de la autoformación. Ambas estrategias son acumulativas de diferentes maneras, incrementando el alcance y la flexibilidad de agentes humanos a través de sus campos de actividad. La hibridez parcial es parte del proceso de manejar la multiplicidad. Sin la hibridez, múltiples identidades se viven como fragmentación y/o contradicción (ver la discusión de las ideas de Baumeister sobre el "déficit de identidad" y el conflicto de identidad en Leong y Ward, 2000: 764). A la inversa, el yo híbrido nuevo debe adaptarse, en cierta medida, entre múltiples entornos. La diferencia entre las dos estrategias nunca es absoluta y se presentan interacciones continuamente, ni la multiplicidad ni la hibridez implican desplazar o renunciar a elementos de la previa identidad. El desplazamiento de identidad es 
una estrategia diferente de formación del sujeto, una que normalmente se impone a los estudiantes desde fuera del yo. Doherty y Singh (2005) describen el desplazamiento de identidad en ciertos programas de integración ${ }^{2}$ para estudiantes internacionales en Australia donde se garantiza la estrategia al buscar la esencia en las diferencias culturales e imponiéndoles un plan de estudios anglo-australiano "puro" que elimina cualquier posibilidad de múltiples afiliaciones pedagógicas y proyectos híbridos.

\section{El yo céntrico}

Se requiere un fuerte sentido de su propio proyecto para maquinar la multiplicidad y la hibridez de manera deliberada. La noción de libertad de agencia de Sen sugiere una voluntad activa, que dé forma y coordine un yo céntrico que sostiene una identidad cambiante mientras gestiona la pluralidad cultural; ese yo céntrico arbitra tensiones y conflictos entre papeles, entre sitios y entre las expectativas de diferentes grupos, propulsa al estudiante hacia encuentros sociales activos con otros, toma decisiones difíciles y cambia de rumbo cuando sea necesario. El yo céntrico no es un individuo entero, delimitado en sí: una identidad con una "I" mayúscula. Es una estación en difusión y cambiante, no un sistema operativo. Sólo es una parte del yo.

La psicología multicultural identifica esta voluntad centrada, coordinante -por un nombre u otro-, como una pieza clave del rompecabezas. Por ejemplo, "la investigación multicultural ha demostrado una asociación estable entre un punto interno de control y el bienestar y la satisfacción psicológica, independientemente del origen de los residentes temporales, inmigrantes o refugiados" (Ward, et al., 2004: 138). Savicki y sus colegas (Savicki, Downing-Burnette, Heller, Binder y Suntinger, 2004) enfatizan elementos positivos, que construyen la agencia en la adaptación intercultural. Chirkov y sus colegas (2007) se enfocan directamente en la autodeterminación. Pyvis y Chapman (2005: 23) encuentran "un nexo de pertenencia múltiple donde definimos quienes somos por las maneras en las que conciliamos nuestras diferentes formas de identidad en una sola identidad". Según Kettle (2005: 48), la agencia es el proceso de producir el yo, el "lugar de múltiples subjetividades". Kettle analizó el caso de un estudiante tailandés quien "trabajaba como agente de su propio cambio" (2005: 45). La agencia no le fue entregada, tuvo que ser autoalimentada.

La investigación multicultural también identifica ciertas cualidades personales que facilitan las funciones centralizadoras de la agencia. Existen estudios que hacen hincapié en la comunicación abierta y directa (Yang, et al., 2006: 490), aprender rápidamente, iniciar y responder, entrar en la imaginación del otro de manera empática

\footnotetext{
${ }^{2}$ Foundation Programs impartidos por instituciones de educación superior al principio de la estancia de los estudiantes internacionales para prepararlos en la educación superior de la institución del país anfitrión, una suerte de programa de integración.
} 
y relacionarse con grupos de cultura/identidad diversa; algunos identifican la regulación emocional, la apertura, la flexibilidad y el pensamiento crítico (Matsumoto, LeRoux, Bernhard y Gray, 2004; Savicki, et al., 2004: 312-313). Bradley (2000: 419) sugiere que algunos estudiantes sobrellevan las cosas bien porque "cargan dos mundos consigo, en sus conjuntos conocidos de comportamientos y percepciones del yo", están especialmente conscientes de la historia de su identidad y esto facilita la autoformación reflexiva. Redmond (2000: 153) habla del "descentramiento social", la capacidad de asimilar los pensamientos y sentimientos de otra persona en todas las culturas. Allan (2003: 83) designa la capacidad de relacionarse con personas independientemente de su origen cultural y usar los diversos encuentros culturales para mejorar la identidad cultural personal. Kashima y Loh (2005) muestran que estudiantes con mayor tolerancia hacia la ambigüedad y la complejidad cultural presentan niveles más elevados de "adaptación". El estudio de Cannon (2000: 364-365) sobre estudiantes indonesios descubre que aprenden a tolerar y entender puntos de vista divergentes: "En términos psicológicos, se han vuelto más complejos, resultado de dos procesos extensos: la diferenciación y la integración”, lo que confirma la multiplicidad y la hibridez. Eventualmente, los estudiantes pasan a un "tercer lugar" que comparten con otros residentes temporales con experiencia, "el punto no limitado de intersección donde los interactuantes de diferentes orígenes culturales y lingüísticos se reúnen y comunican entre ellos exitosamente" (Cannon, 2000: 373).

Muchos investigadores argumentan que la competencia comunicativa, o facilidad en las relaciones multiculturales, son condiciones efectivas de agencia. El estudiante tailandés de Kettle (2005: 51) creía que su agencia efectiva no existía hasta que aprendió a comunicarse e interactuar con personas locales. Lo contrario también aplica: la agencia fuerte ayuda tanto en el dominio de un idioma como en las relaciones multiculturales. Li y Gasser (2005) se centran en la autoeficacia multicultural, combinando interactividad con agencia. Ward y sus colegas (2004) discuten la extraversión, combinando la comunicación y agencia. Mientras más amplia es el área de interacción entre estudiantes internacionales y anfitriones locales, más oportunidades hay para la fabricación creativa de identidades. La discriminación obliga a la división y al conflicto de identidad en lugar de la unión de la identidad (hibridez) o el manejo de multiplicidad bajo el control del estudiante internacional (Leong y Ward, 2000: 771). Esta interdependencia a tres vías: agencia, comunicación e interacción multicultural son recurrentes a través de la investigación, y se confirma de nuevo en Marginson et al. (2010). No obstante, muchos estudiantes que no dominan completamente el inglés o no tienen contacto cercano con las personas locales, presentan un fuerza autónoma extraordinaria. El estrés de la estancia en el extranjero, mientras agota energías e imaginaciones para el que la vive, sugiere que las personas son robustas, no personas frágiles atrapadas por el conflicto cultural. Aun cuando los estudiantes son subordinados por prácticas etnocéntricas que los colocan en una posición de déficit, su sentido del yo es suficientemente fuerte como 
para adaptarse a esas prácticas y, al mismo tiempo, manejan sus propias reacciones emocionales a ser despojadas de estatus.

\section{Conclusiones}

La educación internacional transfronteriza no se define como otra-formación sino como autoformación, en la que los sujetos-estudiantes manejan sus propias vidas y continuamente dan forma a su yo, en cambios constantes. El estudiante debe crear un yo -es decir, la constelación de percepciones, intenciones, recuerdos, valores, hábitos y acciones, incluyendo un sentido de lo que es importante- en un mundo de identidades plurales: país de origen, país anfitrión y tal vez un conjunto más amplio de opciones cosmopolitas. La idea de la autoformación también se centra en la direccionalidad interior, la voluntad propia. Un periodo prolongado de educación transfronteriza exige un mayor esfuerzo por parte de estudiantes internacionales provenientes de países donde no se habla el inglés. Requiere una capacidad avanzada en agencia personal.

El yo y la trayectoria del estudiante internacional se crean continuamente en una cambiante combinación de (1) condiciones materiales, (2) relaciones sociales en las que el estudiante se integra y (3) la libertad de agencia o voluntad activa del estudiante. Cada estudiante enfrenta muchos desafíos y problemas, ninguno es totalmente dueño de su destino, como ninguno de nosotros lo somos, pero desde la perspectiva de la autoformación la agencia consciente del estudiante es irreducible y omnipresente. Un énfasis en la agencia activa apunta a diferentes observaciones y hallazgos con respecto a los que resultan cuando los estudiantes transfronterizos son colocados en un marco de estrés y enfrentamiento, con énfasis en la disfunción o el déficit de bienestar, como se encuentra en mucha de la literatura psicopedagógica (Ward y Rana-Deuba, 1999: 423-424). La perspectiva de la autoformación sugiere que las estrategias pedagógicas que niegan la autoformación tienen propósitos cruzados sobre el aprendizaje real que se lleva a cabo; hace notar las estrategias usadas y las decisiones tomadas por los estudiantes en el país anfitrión, incluyendo las herramientas y los recursos que utilizan en la construcción de ambientes y en la toma de decisiones proactivas. La intención no es menospreciar la necesidad de los servicios proporcionados para las necesidades específicas de los estudiantes internacionales, sino para argumentar que las soluciones a menudo se encuentran en fortalecer la libertad de agencia y su alcance. La ampliación del espacio, donde los estudiantes están libres de constricciones y coacciones -por ejemplo, administraciones autoritarias o prácticas discriminatorias-, aumenta la libertad como control. Mejorando los recursos que facilitan la agencia -por ejemplo, programas diseñados para aumentar competencias comunicativas o proporcionar viviendas- aumenta la libertad como poder. 
La educación superior como autoformación es, a la vez, un marco normativo y una realidad existente. Se puede observar empíricamente en los salones de clases y en las vidas de los estudiantes. También es un enfoque distinto a la educación internacional que se basa en la autodeterminación reflexiva de sujetos-estudiantes. Las instituciones y los profesores pueden desarrollar la agencia consciente de estudiantes internacionales y trabajar con ella, en lugar de sobornarla o forzarla. La enseñanza, como los servicios estudiantiles y la organización institucional, puede promover a los estudiantes internacionales $-y$ sus historias, identidades, perspectivas, prácticas de aprendizaje y decisiones- a que sean dignos del mismo respeto. Esa es la clave de la ruptura decisiva del etnocentrismo. Después de esto, el encuentro del estudiante internacional con un sistema de educación superior en inglés se entiende no como un viaje de conversión sino como una negociación cultural que nunca termina.

El enfoque adoptado en este artículo es desafiante, pero consta del apoyo de otros académicos (Asmar, 2005: 293; Singh, 2005: 10). Volet y Jones (2012: resumen) discuten "el papel descuidado de la agencia en la investigación de la adaptación sociocultural de los estudiantes internacionales". Como se ha señalado, este trabajo no proporciona pruebas finales sobre la autoformación, pues esto requiere de pruebas empíricas; sin embargo, ha sido apoyado por algunos sujetos-estudiantes. Cuando la idea de la educación internacional como autoformación se discute en los seminarios impartidos por el autor con los estudiantes del este y el sureste asiático, una respuesta común es la sorpresa de reconocerse inesperadamente con el contenido del seminario: “¡Sí, eso es! ¡Eso soy!”, declaran los estudiantes. “Sí, eso es lo que estoy haciendo aquí".

\section{Referencias}

Allan, M. (2003). Frontier crossings: Cultural dissonance, intercultural learning and the multicultural personality. Journal of Research in International Education, 2(1), 83-110.

Appadurai, A. (1996). Modernity at large: Cultural dimensions of globalization. Minneapolis: University of Minnesota Press).

Asmar, C. (2005). Internationalizing students: Reassessing diasporic and local student differences. Studies in Higher Education, 30 (3), 291-309.

Ballard, B. \& Clanchy, J. (1991). Teaching students from overseas: A brief guide for lecturers and supervisors. Melbourne: Longman Cheshire.

Berry, J. (1974). Psychological aspects of cultural pluralism. Topics in Cultural Learning, 2, 17-22.

Bochner, S. (1972). Problems in culture learning. In S. Bochner \& P. Wicks (Eds), Overseas students in Australia. Sydney: University of New South Wales Press.

Bourdieu, P. (1993). The field of cultural production. New York: Columbia University Press.

Bradley, G. (2000). Responding effectively to the mental health needs of international students. Higher Education, 39, 417-433. 
Butcher, A. (2002). A grief observed: Grief experiences of East Asian students returning to their countries of origin. Journal of Studies in International Education, 6 (4), 354-368.

Cannon, R. (2000). The outcomes of an international education for Indonesian graduates: The third place? Higher Education Research and Development, 19(3), 357-379.

Church, A. (1982). Sojourner adjustment. Psychological Bulletin, 91 (3), 540-572.

Chirkov, V., Vansteenkiste, M., Tao, R. \& Lynch, M. (2007). The role of self-determined motivation and goals for study abroad in the adaptation of international students. International Journal of Intercultural Relations, 31, 199-222.

Doherty, C. \& Singh, P. (2005). How the West is done: Simulating Western pedagogy in a curriculum for Asian international students. In: P. Ninnes \& M. Hellsten (Eds.), Internationalizing higher education: Critical explorations of pedagogy and policy. Dordrecht: Springer.

Goldbart, J., Marshall, J, \& Evans, I. (2005). International students of speech and language therapy in the UK: Choices about where to study and whether to return, Higher Education, 50, 89-109.

Hofstede, G. (1998). Think locally, act globally: Cultural constraints in personal management. Management International Review, 38 (2), 7-26.

Kashima, E. \& Loh, E. (2006). International students' acculturation: Effects of international, conational, and local ties and need for closure. International Journal of Intercultural Relations, 30, 471-485.

Kettle, M. (2005). Agency as discursive practice: From 'nobody' to 'somebody' as an international student in Australia. Asia-Pacific Journal of Education, 25 (1), 45-60.

Lee, I. \& Koro-Ljunberg, M. (2007). A phenomenological study of Korean students' acculturation in middle schools in the USA. Journal of Research in International Education, 6 (1), 95-117.

Lee, J. \& Rice, C. (2007). Welcome to America? International student perceptions of discrimination. Higher Education, 53, 381-409.

Leong, C. \& Ward, C. (2000). Identity conflict in sojourners. International Journal of intercultural Relations, 24, 763-776.

Li, A. \& Gasser, M. (2005). Predicting Asian international students' sociocultural adjustment: A test of two mediation models. International Journal of Intercultural Relations, 29, 561-576.

Marginson, S. (2012). Including the Other: Regulation of the human rights of mobile students in a nation-bound world. Higher Education, 63 (4), 497-512.

Marginson, S., Nyland, C., Sawir, E. \& Forbes-Mewett, H. (2010). International student security. Cambridge: Cambridge University Press.

Marginson, S. \& Sawir, E. (2011). Ideas for intercultural education. New York: Palgrave Macmillan.

Matsumoto, D., LeRoux, J., Bernhard, R. \& Gray, H. (2004). Unraveling the psychological correlates of intercultural adjustment potential. International Journal of Intercultural Relations, 28, 281-309.

Montgomery, C. (2010). Understanding the international student experience. London: Palgrave Macmillan.

Pedersen, P. (1991). Counselling international students. The Counselling Psychologist, 19 (10), 10-58.

Pyvis, D. \& Chapman, A. (2005). Culture shock and the international student 'offshore'. Journal of Research in International Education, 4 (1), 23-42.

Ramia, G., Marginson, S, \& Sawir, E. (2013). Regulating students' wellbeing. Bristol: The Policy Press. 
Ramsay, S., Barker, M. \& Jones, E. (1999). Academic adjustment and learning processes: A comparison of international and local students in first-year university. Higher Education Research and Development, 18 (1), 129-144.

Redmond, M. (2000). Cultural distance as a mediating factor between stress and intercultural competence. International Journal of Intercultural Relations, 24, 151-159.

Rizvi, F. (2005). Identity, culture and cosmopolitan futures. Higher Education Policy, 18, 331339.

Rizvi, F. (2008). Epistemic virtues and cosmopolitan learning. The Australian Educational Researcher, 35 (1), 17-35.

Rizvi, F. (2009). Towards cosmopolitan learning. Discourse, 30 (3), 253-268.

Rose, N. (1999). Powers of freedom: Reframing political thought. Cambridge: Cambridge University Press.

Savicki, V., Downing-Burnette, R., Heller, L., Binder, F. \& Suntinger, W. (2004). Contrasts, changes and correlates in actual and potential intercultural adjustment. International Journal of Intercultural Relations, 28, 311-329.

Sawir, E., Marginson, S., Deumert, A., Nyland, C. \& Ramia, G. (2008). Loneliness and international students: An Australian study. Journal of Studies in International Education, 12 (2), 148-180.

Sen, A. (1985). Well-being, agency and freedom: The Dewey Lectures 1984. The Journal of Philosophy 82 (4), 169-221.

Sen, A. (1992). Inequality re-examined. Cambridge: Harvard University Press.

Sen, A. (1999). Global justice: Beyond international equity. In: I. Kaul, I. Grunberg \& M. Stern (Eds.), Global public goods: International cooperation in the 21st century, 116-125. New York: Oxford University Press.

Sen, A. (2000). Development as freedom. New York, Anchor Books.

Singh, M. (2005). Enabling translational learning communities: Policies, pedagogies and politics of educational power. In: P. Ninnes \& M. Hellsten (Eds.), Internationalizing higher education: Critical explorations of pedagogy and policy. Dordrecht: Springer.

Volet, S. \& Jones, C. (2012). Cultural transitions in higher education: Individual adaptation, transformation and engagement. In S. Karabenick \& T. Urdan (Eds.) Transitions across schools and cultures. Advances in motivation and achievement, Volume 17 (pp. 241-284). Bingley: Emerald Group Publishing Limited.

Volet, S. \& Ang, G. (1998). Culturally mixed groups on international campus: An opportunity for inter-cultural learning. Higher Education Research \& Development, 17 (1), 5-24.

Ward, C. \& Chang, W. (1997). 'Cultural fit': A new perspective on personality and sojourner adjustment. International Journal of Intercultural Relations, 21 (4), 525-533.

Ward, C., Leong, C. \& Low, M. (2004). Personality and sojourner adjustment: An exploration of the Big Five and the cultural fit proposition. Journal of Cross-Cultural Psychology, 35 (2), 137-151.

Ward, C. \& Rana-Deuba, A. (1999). Acculturation and adaptation revisited. Journal of CrossCultural Psychology, 30 (4), 422-442.

Yang, R., Noels, K. \& Saumure, K. (2006). Multiple routes to cross-cultural adaptation for international students: Mapping the paths between self-construals, English language confidence, and adjustment. International Journal of Intercultural Relations, 30, 487-506. 